\title{
Long live the alien: is high genetic diversity a pivotal aspect of crested porcupine (Hystrix cristata) long-lasting and successful invasion?
}

\author{
Emiliano Trucchi ${ }^{1,2 *}$, Benoit Facon ${ }^{3}$, Paolo Gratton ${ }^{4}$, Emiliano Mori $^{5}$, Nils Chr. Stenseth ${ }^{1}$, Sissel \\ Jentoft ${ }^{1,6}$ \\ ${ }^{1}$ Centre for Ecological and Evolutionary Synthesis, Department of Biosciences, University of Oslo, P.O. Box 1066 Blindern NO- \\ 0316, Norway \\ ${ }^{2}$ Division of Systematics and Evolutionary Botany, Department of Botany, University of Vienna, Rennweg 14, 1030, Vienna, Austria \\ ${ }^{3}$ INRA, UMR1062, CBGP, Avenue du Campus Agropolis, 34980 Montferrier-sur-Lez \\ France \\ ${ }^{4}$ Department of Primatology, Max Planck Institute for Evolutionary Biology, Deutscher Platz 6, 04130, Leipzig, Germany \\ ${ }^{5}$ Department of Agriculture, Forest and Food Science, University of Turin, L.go P. Braccini 2, 10095, Grugliasco, Italy \\ ${ }^{6}$ Department of Natural Sciences, University of Agder, 4604 Kristiansand, Norway \\ *emiliano.trucchi@univie.ac.at
}

Keywords: Invasive species, Population dynamics, Inbreeding, RAD sequencing, Gene surfing.

\begin{abstract}
Studying the evolutionary dynamics of an alien species surviving and continuing to expand after several generations can provide fundamental information on the relevant features of clearly successful invasions. Here, we tackle this task by investigating the dynamics of the genetic diversity in invasive crested porcupine (Hystrix cristata) populations, introduced to Italy about 1500 years ago, which are still growing in size, distribution range and ecological niche. Using genome-wide RAD markers, we describe the structure of the genetic diversity and the demographic dynamics of the $H$. cristata invasive populations and compare their genetic diversity with that of native African populations of both $H$. cristata and its sister species, $\mathrm{H}$. africaeaustralis. First, we demonstrate that genetic diversity is lower in both the invasive Italian and the North Africa source range relative to other native populations from Sub-Saharan and South Africa. Second, we find evidence of multiple introduction events in the invasive range followed by very limited gene flow. Through coalescence-based demographic reconstructions, we also show that the bottleneck at introduction was mild and did not affect the introduced genetic diversity. Finally, we reveal that the current spatial expansion at the northern boundary of the range is following a leading-edge model characterized by a general reduction of genetic diversity towards the edge of the expanding range. We conclude that the level of genome-wide diversity of $\mathrm{H}$. cristata invasive populations is less important in explaining its successful invasion than species-specific life-history traits or the phylogeographic history in the native source range.
\end{abstract}

\section{Introduction}

One of the most relevant and debated questions in invasive biology concerns the importance of standing genetic diversity for successful invasions and colonization of a novel range (Reed \& Frankham 2003, Frankham 2004, Facon et al. 2006, Roman \& Darling 2007). In addition to the initial bottleneck at introduction, which may (Schmid-Hempel et al. 2007, Dlugosch \& Parker 2008, Ciosi et al. 2008, Chapple et al. 2013) or may not (Kolbe et al. 2004, Roman \& Darling 2007, Estoup \& Guillemaud 2010, Hufbauer et al. 2013) decrease the genetic diversity of the introduced propagule, subsequent range expansion can also negatively affect diversity (Edmonds et al. 2004, White et al. 2013), thus likely limiting the adaptive potential of invasive populations and, ultimately, their further spread and/or persistence (Shine et al. 2011). Nevertheless, low neutral genetic diversity of the invasive species per se does not necessarily result in reduced adaptive capability (Dlugosch \& Parker 2008). Rapid genetic adaptation in response to changed selective pressures encountered in the novel environment has been suggested as a possible explanation of very successful colonization events (Prentis et al. 2008) and, in a few cases, fast genetic changes in relevant genes have been discovered (Vandepitte et al. 2014). Indeed, past investigations have often reported cases of successful biological invasions despite low genetic diversity (e.g. Lavergne \& Molofsky 2007, Hardesty et al. 2012). As such, other factors related to ecological traits of the exotic species and/or of the invaded ecosystem and coincidental events may be more important than initial genetic diversity in determining the success of an invasive population (Zayed et al. 2007). However, the vast majority of studies of biological invasions have so far utilized systems with a recent history of introduction, and thus lack a deeper temporal perspective (Strayer et al. 2006) leaving us with a dearth of assessments of the long-term adaptive potential of successful biological invaders (but see Cooling et al. 2011).

The crested porcupine, Hystrix cristata, was historically introduced to Italy (Italian peninsula and Sicily), making it an excellent study system to test the importance of initial genetic diversity for an invasive population to persist and spread. Ancient Romans very likely brought this animal from North Africa as an exotic pet for their villas and as a delicacy for their banquets: a genetic survey based on three mitochondrial genes identified the most likely source in North Africa (i.e. Tunisia) and estimated the introduction event between 2500 and 1500 years ago (Trucchi \& Sbordoni 2009). Independent analyses of archaeological evidence and iconographic documentation suggested that the 
introduction occurred in late Antiquity or the early middle ages (1500-1200 years ago) and that the species' presence in Sicily is not supported before early modern times (Masseti et al. 2010). Nevertheless, we expect a lag time between the accidental release of captive animals and their spread into the invasive range, i.e. when it would have been common enough to be found in archaeological sites. Based on this, we can assume that the introduction of $H$. cristata into peninsular Italy likely started around 1500 years ago, and its populations are viable and still expanding after several hundred generations. In this species, sexual maturity is achieved at the age of ca. 1 year and the following inter-litter interval is about 91112 days (Mohr 1965, Weir 1974).

Records of the distribution of the porcupine in Italy reveal a dramatic range expansion in the last 4050 years (Angelici \& Amori 1999, Mori et al. 2013). During this period, the invasive population crossed the Apennines and colonized the eastern side of the Italian peninsula, passing the Po river in the Padana plain and getting as far north as the southern edge of the Alps. Intriguingly, the newly colonized area is climatically distinct from the pre-expansion range: warm temperate continental climate vs. Mediterranean coastal climate (Blasi et al. 2014). As the former climate type is not present in the source area (North Africa), this sudden range shift may have been driven by a novel adaptation. In stark contrast with the range expansion in North Italy, the extant populations in North Africa are currently declining due to intense anthropic pressure (Saleh \& Basuony 1998; Nowak 1999; Cuzin 2003; Mohamed 2011) and the ongoing aridification of the region (Thuiller et al. 2006; Kröpelin et al. 2008). Additionally, the species still commonly occurs in Sub-Saharan Africa, from Senegal to Ethiopia and Tanzania. A sibling species, H. africaeaustralis (Cape porcupine), is found in austral Africa, from the Democratic Republic of Congo and Tanzania to South Africa (Nowak 1999). These two sister species are phylogenetically and ecologically very close (Mohr 1965, Trucchi \& Sbordoni 2009) and their ranges of distribution largely overlap in East Africa, meaning that $H$. africaeaustralis can be used as an excellent comparison with $H$. cristata for assessing the level of genetic diversity in native populations

Employing a vast RAD sequencing dataset of more than 30,000 loci, we describe the genetic structure and diversity of the invasive Italian $H$. cristata population and compare it to the populations of $H$. cristata and $H$. africaeaustralis found in Africa. We also investigate the smaller scale genetic pattern of the Italian population that is currently expanding northwards. Finally we assess how the introduction and expansion processes have affected the genetic diversity along the whole colonization trajectory, and investigate whether high genome-wide diversity was, and still is, an important aspect of $H$. cristata's successful invasion.

\section{Methods \\ Sampling}

A total of $280 \mathrm{H}$. cristata samples (244 from Italy and 36 from North-Central Africa) and $43 \mathrm{H}$. africaeaustralis samples from Southern Africa were collected in the fieldbetween 2004-2012 from Egypt, Ivory Coast, Ghana, Nigeria, Tanzania, Mozambique, Zambia and Namibia. Most of the samples were quills collected on the ground, but 8 of them (of $H$. cristata from Italy) were from muscle tissue (roadkill). Age and quality of preservation varied greatly across samples. DNA was extracted using the DNAse Blood and Tissue kit (Qiagen) following manufacturer's instructions. Quality and quantity of DNA was checked to identify high-quality samples suitable for genomic analysis. Concentration of DNA was measured using a fluorimetric method (Qubit, Invitrogen) and quality was checked using a spectroscopic method (Nanodrop) and by visual inspection of degradation after gel electrophoresis. Given the uneven quality and DNA preservation of the sampled materials, ca. $80 \%$ of the samples were discarded as unsuitable for genomic analyses because of strong DNA degradation, and ca. $10 \%$ of the remaining samples failed in the sequencing run.

A total of 50 samples of $H$. cristata (38 from the invasive Italian populations and 12 from native African populations) and 11 samples of $H$. africaeaustralis were selected for RAD sequencing (Fig. 1, Supplementary Table 1). Samples were grouped according to species (H.cristata: H.cri; $H$. africaeaustralis: H.afr) and geographical origin (H.criSS: Sub-Saharan Africa; H.cri-NA: North Africa; H.cri-IT-Sicily: Sicily, Italy; H.cri-IT-south: South Italy; H.cri-IT-centre: Central Italy; H.cri-IT-north: North Italy). In addition, samples in H.cri-IT-north were further grouped according to the year of establishment of a population (Angelici et al. 2003, Mori et al. 2013) in the area they were sampled (H.criIT-north-1959: distribution in 1959; H.cri-IT-north1999: distribution in 1999; H.cri-IT-north-2012: distribution in 2012).

\section{RAD sequencing}

The RAD sequencing protocol from Baird et al. (2008) was slightly modified to prepare the libraries. Approximately $100 \mathrm{ng}$ of genomic DNA per sample were digested with the restriction enzyme $S b f$ I (NEB). Each sample was ligated to a unique barcoded P1 adapter prior to pooling in a single library. Libraries were sheared by sonication on a Bioruptor (Diagenode) where the target size range fraction of 300-500 bp was achieved after seven cycles of sonication (30 seconds ON, 30 seconds OFF). After concentration to $25 \mu$ l by DNA capture on magnetic beads (beads solution : DNA $=0.8: 1$ ), libraries were 
size selected by gel electrophoresis and manual excision. Capture on magnetic beads (beads solution : DNA $=0.8: 1$ ) was then employed in all following purification steps (i.e. after blunt-end repairing, poly-A tailing, P2 adapter ligation and library enrichment by PCR). To reduce amplification bias, PCR reactions were split in $8 \times 12.5 \mu$ l aliquots per library, separately amplified and then pooled again. Libraries were quantified by a fluorimetric-based method (Qubit, Invitrogen) and molarity was checked on an Agilent Bioanalyzer chip (Invitrogen). A final volume of ca. 20 $\mu \mathrm{l}$ per library with a DNA concentration of 20-25 ng/ $\mu \mathrm{l}$ was submitted for paired-end $100 \mathrm{bp}$ sequencing (two lanes on a ILLUMINA HiSeq2000) at the Norwegian Sequencing Centre, University of Oslo.

\section{Bioinformatic analyses}

Raw reads were processed using the scripts included in the Stacks package (Catchen et al. 2013) on the ABEL cluster server facility at the University of Oslo. Raw reads were quality filtered and demultiplexed according to individual barcodes using the script process_radtags.pl in the Stacks package with default settings. Cleaned reads were then aligned into loci and SNPs called across individuals using the script denovo_map.pl in the Stacks package: the minimum coverage to call a stack of identical reads was set to 10 (option -m), the maximum number of mismatches allowed when joining stacks into the same locus to 7 (option -n) and the maximum number of mismatches allowed when joining loci across individuals to 7 (option -N). In order to capture different levels of genetic variability within and between populations of the two species, we built three separate catalogs: $i$ ) one catalog including all Italian and North Africa individuals (invasive dataset, 42 individuals), ii) one catalog including a subset of the Italian individuals taking into account both an even representation of the invasive range and the highest average coverage across genotyped loci (invasive_reduced dataset, 16 samples: H.cri-IT; Fig. 1), and iii) one catalog including the same subset of high-coverage Italian individuals as in the previous catalog as well as all of the African individuals (global dataset, 39 individuals). We included only a small subset of the invasive samples (16) in the global catalog in order to avoid overweighting the genetic diversity in the invasive population, where the largest proportion of samples were analyzed, and to minimize the SNPs ascertainment bias in this dataset as a result. The function export_sql.pl in the Stacks package was used to extract loci information from each catalog applying a global threshold of $25 \%$ to the maximum number of missing sample per locus and 10 to the maximum number of SNPs per locus. This high threshold for the maximum number of SNPs per locus, especially in the case of the invasive population, was justified by the consideration that filtering loci on the basis of their shallow variation might introduce a severe bias towards recent coalescent events. In the case of the invasive population, the only possibility to retrieve the genetic signature of a bottleneck is to find divergent alleles whose coalescence time is deeper than the bottleneck itself and that can provide information about the pre-bottleneck ancestral population size. In addition, the accumulation of substitutions along the genome is a random process with a mean rate and extreme cases are expected. A careful screening of putative paralogous loci or non-random distribution of SNPs along each locus was then performed as follows. We wrote custom python scripts (see Supplementary Materials) to further filter the dataset in order to exclude loci with more than 2 alleles per individual, with heterozygosity above 0.75 , or deleveraged by the Stacks algorithm. We found an increase in the number of SNPs called in the last 10 positions across all of the loci (Fig. S1); trimming the raw reads of $10 \mathrm{bp}$ and performing again the SNP calling produced the same pattern. We believe this is an artifact of the SNP calling algorithm, so any SNP recorded in the last 10 base pairs of each locus was considered unreliable and discarded. Further filtering to reduce missing data was applied on a case-by-case basis, according to each downstream analysis.

\section{Genetic structure of native and invasive populations}

The global dataset was used to infer the overall structure among native and invasive populations of the two porcupine species. Following Wagner et al. (2013), loci were concatenated into a single sequence per sample, coding heterozygous sites as ambiguities in agreement with the IUPAC code. The whole sequence of each locus was included in order to get empirical estimates of base composition and percentage of invariant sites. A Maximum-Likelihood algorithm with a GTR + G + I substitution model was employed to reconstruct the phylogenetic tree of our samples using 100 rapid bootstrap inferences and thereafter a thorough ML search in RAxML 7.2.8 (Stamatakis 2006). Results were visualized and edited in FigTree

1.4

(http://tree.bio.ed.ac.uk/software/figtree/). Although this cannot be considered a true phylogenetic reconstruction, this random concatenation of recombining genomic fragments has proven to be informative (Wagner et al. 2013).

The invasive dataset was then used to study the fine-scale geographic structure of the $H$. cristata invasive population and its relationship with North Africa source population. First, using all of the SNPs in each locus and all of the samples in the dataset (H.cri-IT and H.cri-NA; Fig. 1), we performed a Neighbor-Joining Network (NeighborNet) analysis (Bryant \& Moulton 2004) based on uncorrelated $p$ distances in Splitstree (Huson \& Bryant 2006). We then performed a Principal Component Analysis 
(PCA) reducing our dataset to only one randomlychosen SNP per locus. To better capture the variance in the invasive range, only individuals belonging to the invasive population (H.cri-IT; Fig. 1) were included in the PCA, while individuals from North Africa were excluded. Given that PCA is very sensitive to missing data (in this case, missing loci in each individual), we further filtered our dataset, removing four individuals with more than 50\% missing loci (EMR01, ITA09, SSC01, LRM03). The strict filtering in this second analysis was necessary because samples with too many missing loci tend to be unassigned (e.g. appear at the centre of the axes). The glPca function in the $R$ packages "adegenet" was used for calculations.

\section{Demography of the invasive population}

We used RAD loci genotyped in the high-coverage Italian individuals (H.cri-IT; Fig.1) to reconstruct the demographic history of the invasive population following an approach recently proposed by Trucchi et al. (2014) that has been proven to be particularly efficient in reconstructing recent demographic events (Tutorial available

at http://www.emilianotrucchi.it/images/EBSP_RADseq_ tutorial.pdf). In short, a selection of highly variable RAD loci with more than 3 SNPs per locus are used as short sequences in a coalescent-theory based multilocus analysis (Extended Bayesian Skyline plot; Heled \& Drummond 2008) implemented in BEAST 1.7.4 (Drummond \& Rambaut 2007). Four random selections of 50 loci with 4 to 9 SNPs (no loci with more than 9 SNPs passed our filtering criteria) from the invasive_reduced dataset were used in four replicated runs and checked for convergence. Analyses were performed as follows: i) nucleotide substitution models, clock models and tree prior models were unlinked across loci; ii) the nucleotide substitution model was set as a HKY with empirical base frequency; iii) a strict molecular clock was set for each marker with a uniform prior distribution of the substitution rate bounded within 0.5 and 0.005 sub/s/Myr; iv) the Extended Bayesian Skyline Plot (EBSP) was selected as a tree prior model. 200 million iterations were set as run length. In addition, we ran the EBSP analyses adding the mtDNA Control Region sequences of the invasive population published in Trucchi \& Sbordoni (2009) to a random selection of 50 RAD loci. We used this analysis to calibrate our demographic reconstruction, applying a substitution rate of 0.2 substitution/site/Myr to the mtDNA marker only and leaving the substitution rates for the RAD loci to be estimated in the analysis. The substitution rate employed for the calibration was derived from the rate estimated in another rodent species (Mus musculus; Rajabi-Maham et al. 2008) and corrected for the longer generation time in porcupines. An HKI with Gamma (4 classes) and Invariant site substitution model and a strict clock model were implemented for the mtDNA Control Region. Three replicates were checked for convergence. All analyses were run on a 24 CPUs server at the University of Oslo. Results were $\begin{array}{lll}\text { checked on } & \text { Tracer }\end{array}$ (http://tree.bio.ed.ac.uk/software/tracer) and the plot of the EBSP data was drawn in $R$ (R Development Core Team 2011). To take into account the structure in the invasive population, we also repeated the EBSP analysis using samples from only one of the groups (H.cri-IT-north).

\section{Heterozygosity estimates}

Events of introduction into a new range are expected to induce a reduction in heterozygosity in the invasive population, and quick range expansions in the invasive range could produce a further clinal reduction in heterozygosity towards the expansion front. Nevertheless, levels of individual heterozygosity ultimately depend on levels of inbreeding (Hoffman et al. 2014), and are influenced by a variety of demographic process at different temporal and spatial scales. Observed and expected heterozygosity were estimated in native and invasive populations/groups of individuals (Fig.1) using the global and the invasive datasets. To test for the effects of the introduction event, we used the global dataset, using the following partition of native and invasive individuals: H.afr, H.cri-SS, H.cri-NA, and H.cri-IT. To test for local effects in the different invasive populations, we used the invasive dataset and the groups H.cri-IT-north, H.cri-IT-central, H.cri-IT-south, H.cri-IT-Sicily, and H.cri-NA. To test for the effects of the recent range expansion in north Italy, we used the invasive dataset and the groups H.cri-IT-north-1959, H.cri-IT-north1999, and H.cri-IT-north-2012.

Despite their relevance as proxies for levels of inbreeding (Slate \& Pemberton 2002), accurate estimates of individual observed heterozygosity from multi-locus data are difficult to calculate (see Aparicio et al. 2006 for a review). We first estimated individual observed heterozygosity as the number of heterozygous loci divided by the number of genotyped loci in each individual $\left(\mathrm{H}_{\mathrm{o}}\right.$ ind $)$. As our RADseq loci were not mapped to a reference genome, a fraction of non-orthologous loci could have been included in the analysis and may appear as loci with artificially high heterozygosity. To address this issue, we downweighted the contribution of each locus according to its observed heterozygosity across individuals $\left(\mathrm{H}_{\mathrm{o}}\right.$ indnorm). To apply this correction, we first calculated the observed locus-by-locus heterozygosity across all of the individuals in the dataset $\left(\mathrm{H}_{0}\right.$ loc $=$ number of heterozygous genotypes / number of genotype at a locus). Then we re-calculated the individual observed heterozygosity correcting the contribution of each locus by a factor $1-\mathrm{H}_{\mathrm{o}}$ loc. Another important aspect to take into account when estimating observed individual heterozygosity from next-generation data is that the 
probability of scoring a heterozygous genotype at a locus is a function of its depth of coverage. To correct for this, we included the coverage depth of each individual as a predictor of individual observed heterozygosity in a linear model and we calculated the deviation of each individual from the model (i.e., analysis of model's residuals). Individual-based residuals were then grouped as described above and compared. An additional analysis using logtransformed values for depth of coverage was also performed to take into account the fact that the probability of retrieving both alleles at a heterozygous locus is expected to reach a plateau. The significance of differences in observed heterozygosity among geographical groups of samples was assessed by F-test comparing a linear model where observed heterozygosity was predicted by both the number of reads and the geographic origin of the sample with a reduced model with the number of reads as the only predictor. When the F-test revealed at least a statistical trend $(\mathrm{P}<0.1)$, the statistical significance of all pairwise contrasts among geographically-defined groups of samples was calculated by running the full linear model $\mathrm{N}-1$ times (with $\mathrm{N}=$ number of groups) and setting, each time, a different group as the reference level for the 'group' factor. Analyses were performed using custom python scripts (available as Supplementary Material) and basic $R$ functions.

Expected heterozygosity (i.e., gene diversity; $\mathrm{H}_{\mathrm{e}}$ ) was also estimated for each of the groups described above according to the formula in Nei (1978). A minimum number of 3 individuals genotyped per group was required to include a locus in the analysis. As compared to $\mathrm{H}_{\mathrm{o}-\mathrm{ind}}, \mathrm{H}_{\mathrm{e}}$ is less biased by the individual sequencing coverage because rare alleles in the population still have $50 \%$ probability to be sequenced in those individuals that carry them. Even if information on the population structure in the native range was scarce, estimation of population-level Watterson's theta and $\pi$ were also calculated for both native and invasive populations. Analyses were performed using custom python scripts (available as Supplementary Material).

\section{Results \\ RAD sequencing data}

After de-multiplexing, the average number of single reads retained per individual was between ca. 500,000 and ca. 7,100,000. The starting quality of the DNA strongly influenced this inequality across individuals, with extractions from muscle tissue resulting in a much higher sequencing yield than extractions from quills. The total number of loci retained in each catalog after filtering was: 17,504 in the invasive_reduced, 30,506 in the invasive and 19,559 in the global dataset with an average coverage per allele per individual of ca. 15X. Distribution of SNPs per position and of individuals genotyped per locus is shown in Figure S2.

\section{Genetic structure of native and invasive populations}

The Maximum-Likelihood tree reconstructed using the information provided by the global dataset showed a clear differentiation between $H$. africaeaustralis and $H$. cristata (Fig. 2); samples belonging to $H$. africaeaustralis formed a cluster of mostly unresolved relationships with little or no geographical structure in the genetic diversity. On the other hand, $H$. cristata populations showed a marked geographic differentiation. In this species, the genetic diversity mirrored its distribution from East Africa (Tanzania and Ethiopia), where $H$. cristata overlaps with $H$. africaeaustralis, to the West (Nigeria, Ivory Coast, Burkina Faso and Senegal), and then northward, to the Mediterranean coast of Africa and Italy (Morocco, Tunisia, Egypt, Italy). In the invasive range, the genetic structure of $H$. cristata samples clearly followed their geographic origin with clusters of individuals corresponding to H.cri-IT-north, H.cri-ITcentre, H.cri-IT-south, and H.cri-IT-Sicily. The latter also included individuals from Tunisia and Egypt (from the group H.cri-NA).

The structure of the genetic diversity in the $H$. cristata invasive range was investigated in more detail using the invasive dataset. Results of the NeighborJoining Network analysis (Fig. 3a) and of the PCA (Fig. $3 b$ ) were consistent with each other and with the Maximum-Likelihood tree estimated on the global dataset. The same four clusters, as described above, clearly characterized the invasive range. Only two individuals did not cluster according to their geographic origin (Fig.1): ITA09 (from the group H.cri-IT-north), sampled at the early stage of the range expansion in the northernmost area occupied in Italy, and MOL02 (from the group H.cri-IT-centre), sampled in central Italy. In all of the analyses, these two samples clustered in H.cri-IT-centre and H.cri-ITnorth, respectively.

\section{Demography of the invasive population}

The reconstruction of the past demography of the invasive population showed a clear signature of a recent, although mild, bottleneck (Fig. 4). According to our result, the coalescent effective population size at introduction corresponded to few hundred individuals, and it was only 5 times lower than before. The time of the bottleneck is estimated to be within the last 2000 years when taking into account the 95\% confidence intervals. The calibrated demography showed a decrease in population size starting well before the putative introduction event, this decrease corresponds to the end of the African Humid Period (AHP), when the green Sahara was turning into a desert (deMenocal et al., 2000). The bottleneck was also found when the RAD loci only and no mtDNA markers were included 
in the analyses (Fig. S3), but it was not detectable when the mtDNA was analyzed on its own (Trucchi \& Sbordoni 2009). Independent runs employing only individuals from H.cri-IT-north produced the same demographic reconstruction, ruling out structure in the invasive population as a source of the observed pattern (not shown).

\section{Heterozygosity estimates}

Estimates of individual observed and population expected heterozygosity are shown in Figure 5 . Robustness of this analysis to the amount of missing data, to the correction for highly-heterozygous loci $\left(\mathrm{H}_{\mathrm{o}}\right.$ ind-norm), and to the log-transformation of the predictor (log-reads) is reported in Figure S4-S5. Considering both observed and expected estimates, heterozygosity was significantly higher $(\mathrm{p}<0.001$ considering all comparisons) in H.afr and H.cri-SS than in H.cri-NA and H.cri-IT (Fig. 5a). In the invasive range, the level of $\mathrm{H}_{\mathrm{o} \text { ind }}$ was similar across all of the groups considered in the analysis $(\mathrm{p}=0.248$ considering all comparisons), only slightly lower in H.cri-IT-south and H.cri-IT-Sicily (Fig. 5b). When the predictor is not logtransformed ( $\mathrm{p}=0.137$ considering all comparisons), $\mathrm{H}_{\mathrm{o}}$ ind in H.cri-NA and H.cri-IT-Sicily was slightly lower and higher than before, respectively (Fig. S5). However, it has to be noted that both groups included only a few individuals (four and three respectively). In contrast to $\mathrm{H}_{\mathrm{o}}$ ind, $\mathrm{H}_{\mathrm{e}}$ was lower in H.cri-IT-south and H.cri-IT-Sicily than in the other groups. When investigating the fate of the heterozygosity along the recent range expansion in H.cri-IT-north (Fig. 5c), our analysis showed a consistent decrease in both observed and expected heterozygosity from the core to the edge of the recently colonized area $(p=0.058$ considering all comparisons). The decrease was particularly evident in the most recently occupied area (H.cri-ITnorth-2012). Mutation-scale effective population size (theta) in the native and invasive range was estimated using the two alternative estimators $\pi$ and Watterson's: 0.27 and 0.30 in H.afr; 0.35 and 0.32 in H.cri-SS; 0.22 and 0.18 in H.cri-NA; 0.22 and 0.20 in H.cri-IT.

\section{Discussion \\ Pivotal drivers of successful invasions}

The $H$. cristata populations introduced in Italy more than 1500 years ago - and still expanding - are the result of an extremely successful invasion (Trucchi \& Sbordoni 2009; Masseti et al. 2010; Mori et al. 2013). Our analyses demonstrate that both genetic diversity, measured as expected heterozygosity $\left(\mathrm{H}_{\mathrm{e}}\right)$, and inbreeding, measured as individual observed heterozygosity $\left(\mathrm{H}_{\mathrm{o}}\right.$ ind $)$, in the invasive populations are lower than in the natural populations of both $H$. cristata and $H$. africaeaustralis from Sub-Saharan and south Africa (Fig. 5a). However, genetic diversity in the native populations of $H$. cristata from the source range in North Africa is similar or slightly lower than in the invasive range. Including only four individuals from different geographic areas, the sample from North Africa is rather small and does not allow us to get conclusive information on the genetic diversity of the source population. Nevertheless, it has to be noted that individuals from Sicily, clustering together with those from Tunisia and Egypt (Fig. 3), show very similar levels of genetic diversity (Fig. 5b) and support the hypothesis that genetic diversity in the source population was lower than in other Sub-Saharan populations. On the other hand, the main finding of our study (i.e. successful invasion and colonization despite low genetic diversity) is supported by the comparison between the invasive population and the two native populations from Sub-Saharan and south Africa. Relative effective population sizes of native and invasive populations, calculated both on the basis of segregating sites and pairwise differences, are consistent with the heterozygosity estimates and also support the conclusion that the introduction bottleneck left a minor, if not negligible, signature on the invasive population. The effective population size in the source population (H.cri-NA) is lower as compared to other native populations (H.cri-SS and H.afr) supporting a past history of contraction and fragmentation in the North Africa range.

Our demographic reconstruction of the invasive populations clearly showed a mild bottleneck (with a few hundred individuals at the lowest point) that can be calibrated at the introduction time (Fig. 4). Nevertheless, the marked genetic structure found in the invasive range (Fig. 3) supports a multiple introduction scenario (in contrast to a single massive introduction and naturalization of hundreds of individuals), where many small propagules were introduced over time by continuous commercial trading instead of . Indeed, Sicily and South Italy could have even been colonized at a later stage as suggested by the higher genetic (Fig. 2, 4) and morphological (Angelici et al., 2003) similarity with North African populations and further supported by the analysis of historical records (Masseti et al. 2010). Interestingly, very limited admixture among different propagules should have characterized the demographic dynamics of the invasive populations in order to retrieve such clear structure today. Thus, increased genetic diversity through admixture in the newly colonized area was not necessary for the long persistence of this invasive species. In such cases, limiting connectivity among different invasive propagules would be of little to no help in preventing the colonization (see Rius and Darling 2014 for a review).

Another important implication of our study is that initial genome-wide diversity does not necessarily explain an invasive species' success. Even if high genetic diversity appears to be positively correlated with success in the early phases of colonization (Forsman 2014), the correlation between genetic 
diversity and long-term viability (and evolvability) of an invasive species could be less straightforward than it intuitively seems (Dlugosh et al. 2015). Indeed, several studies described successful invasions despite an initial low genetic diversity (e.g. Zayed et al. 2007, Harrison \& Mondor 2011) or, alternatively, reporting an increased diversity in the invasive range (e.g. Lavergne \& Molofsky 2007, Signorile et al. 2014). We suggest that a thorough understanding of the phylogeographic history as well as the phenotypic and genomic traits of the source populations is necessary to clearly identify common features of highly invasive species.

In the case of $H$. cristata, introduced individuals were likely sourced from North African populations that already had low genetic diversity. In fact, North African populations were (and still are) suffering from the ongoing desertification of the Sahara, which started approximately 6000 years ago (deMenocal et al. 2000). This long history of habitat fragmentation after the African Humid period is likely to have caused contraction and isolation of North African populations, thus increasing inbreeding and decreasing (local) genetic diversity. One of the shortterm effects of inbreeding is an increased probability that an individual could carry recessive deleterious alleles in homozygosity, decreasing its fitness and, in general, reducing the viability of the small population (Charlesworth \& Willis 2009). Nevertheless, if a small population survives a long period of inbreeding, the expectation is that some (or most) of the deleterious alleles have already been exposed to selection and likely purged from its gene pool (Crow 1970), making that population more resistant to further inbreeding (but see Crnokrak \& Barret 2002 for a review). This has been shown in laboratory experiments where higher fitness was found in invasive populations that experienced mild bottlenecks and high inbreeding in the past compared to native populations that never experienced a bottleneck (Facon et al. 2011, Tayeh et al. 2013). The long history of fragmentation and isolation of $H$. cristata in North Africa could have then favoured the purging of several deleterious alleles in the source gene pool. Individuals originating from these populations could have been less susceptible to the negative effects of small population size at introduction and, as a result, more efficient in establishing a viable population in the invasive range.

\section{Recent invasive evolutionary dynamics}

In this study, we further demonstrate that the dramatic range expansion of $H$. cristata recorded in the last 50 years in Italy is following a leading-edge pattern (Hewitt 1996), with the north population acting as the only colonization source (Fig. 1, 2, 4). One exception in our dataset is the sample collected in North Italy (ITA09), at an early stage of the range expansion, which is genetically similar to the Central Italy population (H.cri-IT-centre). This mismatch could be explained by labeling error, or could actually be the result of long-distance dispersal due to humanmediated translocation of individuals (Mori et al. 2013). The same reasoning could be applied to the sample in Central Italy (MOL02) that clusters with the northern group.

Our analyses reveal a clear decrease in the genetic diversity with the year of colonization (Fig. 5c), mainly as individual observed heterozygosity but also in terms of expected heterozygosity at the northernmost edge of the expansion. Nevertheless, the overall pattern of genetic diversity is consistent with expectations drawn from the gene surfing model (Edmonds et al. 2004, DeGiorgio et al. 2011). According to this model, genetic diversity is reduced at the leading edge of the expansion, whereas local gene flow and admixture is expected to balance the diversity loss at the trailing edge. However, if there is any spatial constraint in the newly colonized area (mountain ridges, rivers), it will be more difficult to reestablish the level of diversity present at the original core of the expansion (Excoffier et al. 2009). The mountain ridge along the Italian peninsula (Apennines) and the main river in the Padana plain (Po) could act as constraints to future gene flow in the expansion range. It remains to be investigated what the main driver of the observed range expansion is. At least three hypotheses can be proposed as an explanation: $i$ ) the reduced anthropic pressure due to the wide-scale abandonment of the countryside after the Second World War and the legal protection of this species since 1980; ii) the effect of ongoing climate change (for analyses of climate change in Italy cf. Brunetti et al. 2006); iii) an emerging adaptation in the north population that allowed the colonization despite relatively novel climate conditions (from Mediterranean sub-coastal to warm temperate areas; Blasi et al. 2014). Based on the solid background about the structure and the dynamics of the neutral genetic diversity provided in this study, further analyses should focus on putative adaptive response(s) in the expanding invasive population, aiming at disentangling the effects of demographic processes from those of selection.

\section{Acknowledgements}

We would like to thank all of the colleagues and friends who kindly helped providing samples from all over Africa. We thank Anna Mazzarella for helpful comments on the early version of the manuscript and four anonymous reviewers for their excellent contribution. This study was supported by Marie Curie Intra European Fellowships (FP7-PEOPLE-IEF-2010, European Commission; project no. 252252 to E.T.) and by the Centre for Ecological and Evolutionary Synthesis, Department of Biosciences, University of Oslo, Norway. 


\section{Data accessibility}

Raw ILLUMINA reads for each sample have been uploaded to the SRA (Acc. num. SRP065809). Processed RAD datasets are publicly available on Dryad repository: doiXXXX

\section{Author contributions}

Designed research: ET, NCS; performed research: ET; contributed analytical tools: ET, PG; contributed sampling ET, EM, PG; analyzed data: ET, PG; wrote the paper: ET, BF, PG, NCS, SJ.

\section{References}

Angelici F.M., \& Amori G. (1999) Distribution of the Crested porcupine Hystrix cristata L., 1758 in peninsular Italy and Sicily. Bollettino del Museo Regionale di Scienze Naturali (Torino), 16, 83-88.

Angelici FM, Capizzi D, Amori G, Luiselli L (2003) Morphometric variation in the skulls of the crested porcupine Hystrix cristata from mainland Italy, Sicily, and northern Africa. Mammalian Biology Zeitschrift für Säugetierkunde, 68, 165-173.

Aparicio JM, Ortego J, Cordero PJ (2006) What should we weigh to estimate heterozygosity, alleles or loci? Molecular Ecology, 15, 4659-4665.

Baird NA, Etter PD, Atwood TS et al. (2008) Rapid SNP Discovery and Genetic Mapping Using Sequenced RAD Markers. PLoS ONE, 3, e3376.

Blasi C, Capotorti G, Copiz R et al. (2014) Classification and mapping of the ecoregions of Italy. Plant Biosystems - An International Journal Dealing with all Aspects of Plant Biology, 148, 1255-1345.

Brunetti M, Maugeri M, Monti F, Nanni T (2006) Temperature and precipitation variability in Italy in the last two centuries from homogenised instrumental time series. International Journal of Climatology, 26, 345-381.

Bryant D, Moulton V (2004) Neighbor-Net: An Agglomerative Method for the Construction of Phylogenetic Networks. Molecular Biology and Evolution, 21, 255-265.

Catchen J, Hohenlohe PA, Bassham S, Amores A, Cresko WA (2013) Stacks: an analysis tool set for population genomics. Molecular Ecology, 22, 31243140.

Chapple DG, Miller KA, Kraus F, Thompson MB (2013) Divergent introduction histories among invasive populations of the delicate skink (Lampropholis delicata): has the importance of genetic admixture in the success of biological invasions been overemphasized? Diversity and Distributions, 19, 134-146.

Charlesworth D, Willis JH (2009) The genetics of inbreeding depression. Nature Reviews Genetics, 10, 783-796.

Ciosi M, Miller NJ, Kim KS et al. (2008) Invasion of
Europe by the western corn rootworm, Diabrotica virgifera virgifera: multiple transatlantic introductions with various reductions of genetic diversity. Molecular Ecology, 17, 3614-3627.

Cooling M, Hartley S, Sim DA, Lester PJ (2011) The widespread collapse of an invasive species: Argentine ants (Linepithema humile) in New Zealand. Biology Letters, rsbl20111014.

Crnokrak P, Barrett SCH (2002) Perspective: Purging the genetic load: A review of the experimental evidence. Evolution, 56, 2347-2358.

Crow JF (1970) Genetic loads and the cost of natural selection In: Mathematical Topics in Population Genetics. Springer, New York.

Cuzin F (2003) Les grands mammifères du Maroc méridional (Haut Atlas, Anti Atlas et Sahara): Distribution, Ecologie et Conservation. Ph.D. Thesis, Laboratoire de Biogéographie et Ecologie des Vertèbrés, Ecole Pratique des Hautes Etudes, Université Montpellier II.

DeGiorgio M, Degnan JH, Rosenberg NA (2011) Coalescence-Time Distributions in a Serial Founder Model of Human Evolutionary History. Genetics, 189, 579-593.

deMenocal P, Ortiz J, Guilderson T et al. (2000) Abrupt onset and termination of the African Humid Period:: rapid climate responses to gradual insolation forcing. Quaternary Science Reviews, 19, 347-361.

Dlugosch KM, Parker IM (2008) Invading populations of an ornamental shrub show rapid life history evolution despite genetic bottlenecks. Ecology Letters, 11, 701-709.

Dlugosch KM, Anderson SR, Braasch J, Cang FA, Gillette HD (2015) The devil is in the details: genetic variation in introduced populations and its contributions to invasion. Molecular Ecology, 24, 2095-2111.

Drummond AJ, Rambaut A (2007) BEAST: Bayesian evolutionary analysis by sampling trees. $B M C$ Evolutionary Biology, 7, 214.

Edmonds CA, Lillie AS, Cavalli-Sforza LL (2004) Mutations arising in the wave front of an expanding population. Proceedings of the National Academy of Sciences of the United States of America, 101, 975979.

Estoup A, Guillemaud T (2010) Reconstructing routes of invasion using genetic data: why, how and so what? Molecular Ecology, 19, 4113-4130.

Excoffier L, Foll M, Petit RJ (2009) Genetic Consequences of Range Expansions. Annual Review of Ecology, Evolution, and Systematics, 40, 481501.

Facon B, Genton BJ, Shykoff J et al. (2006) A general eco-evolutionary framework for understanding bioinvasions. Trends in Ecology \& Evolution, 21, 130-135.

Facon B, Hufbauer RA, Tayeh A et al. (2011) 
Inbreeding Depression Is Purged in the Invasive Insect Harmonia axyridis. Current Biology, 21, 424427.

Forsman A (2014) Effects of genotypic and phenotypic variation on establishment are important for conservation, invasion, and infection biology. Proceedings of the National Academy of Sciences, 111, 302-307.

Frankham R (2004) Resolving the genetic paradox in invasive species. Heredity, 94, 385-385.

Hardesty BD, Le Roux JJ, Rocha OJ et al. (2012) Getting here from there: testing the genetic paradigm underpinning introduction histories and invasion success. Diversity and Distributions, 18, 147-157.

Harrison JS, Mondor EB (2011) Evidence for an Invasive Aphid "Superclone": Extremely Low Genetic Diversity in Oleander Aphid (Aphis nerii) Populations in the Southern United States. PLoS ONE, 6, e17524.

Heled J, Drummond AJ (2008) Bayesian inference of population size history from multiple loci. BMC Evolutionary Biology, 8, 289.

Hewitt GM (1996) Some genetic consequences of ice ages, and their role in divergence and speciation. Biological Journal of the Linnean Society, 58, 247276.

Hoffman JI, Simpson F, David P et al. (2014) Highthroughput sequencing reveals inbreeding depression in a natural population. Proceedings of the National Academy of Sciences, 111, 3775-3780.

Hufbauer RA, Rutschmann A, Serrate B, Vermeil de Conchard H, Facon B (2013) Role of propagule pressure in colonization success: disentangling the relative importance of demographic, genetic and habitat effects. Journal of Evolutionary Biology, 26, 1691-1699.

Huson DH, Bryant D (2006) Application of Phylogenetic Networks in Evolutionary Studies. Molecular Biology and Evolution, 23, 254-267.

Kolbe JJ, Glor RE, Rodríguez Schettino L et al. (2004) Genetic variation increases during biological invasion by a Cuban lizard. Nature, 431, 177-181.

Kröpelin S, Verschuren D, Lézine A-M et al. (2008) Climate-Driven Ecosystem Succession in the Sahara: The Past 6000 Years. Science, 320, 765-768.

Lavergne S, Molofsky J (2007) Increased genetic variation and evolutionary potential drive the success of an invasive grass. Proceedings of the National Academy of Sciences, 104, 3883-3888.

Masseti M, Albarella U, De Grossi Mazzorin J (2010) The crested porcupine, Hystrix cristata L., 1758, in Italy. Anthropozoologica 45, 27-42.

Mohamed WF (2011) The Crested Porcupine Hystrix cristata (Linnaeus, 1758) in Misurata, Libya. European Journal of Biological Sciences, 3, 9-12.

Mohr E (1965) Altweltliche Stachelschweine. A. Ziemsen Verlag, Westarp Wissenschaften (ed),
Wittenburg Lutherstadt, Germany.

Mori E, Sforzi A, Di Febbraro M (2013) From the Apennines to the Alps: recent range expansion of the crested porcupine Hystrix cristata L., 1758 (Mammalia: Rodentia: Hystricidae) in Italy. Italian Journal of Zoology, 80, 469-480.

Nei M (1978) Estimation of average heterozygosity and genetic distance from a small number of individuals. Genetics, 89, 583-590

Nowak RM (1999) Walker's Mammals of the World. The Johns Hopkins University Press, Baltimore and London.

Prentis PJ, Wilson JRU, Dormontt EE, Richardson DM, Lowe AJ (2008) Adaptive evolution in invasive species. Trends in Plant Science, 13, 288-294.

R Development Core Team (2011) $R$ Foundation for Statistical Computing, Vienna, URL http://www.Rproject.org.

Rajabi-Maham H, Orth A, Bonhomme F (2008) Phylogeography and postglacial expansion of Mus musculus domesticus inferred from mitochondrial DNA coalescent, from Iran to Europe. Molecular Ecology, 17, 627-641.

Reed DH, Frankham R (2003) Correlation between Fitness and Genetic Diversity. Conservation Biology, 17, 230-237.

Roman J, Darling JA (2007) Paradox lost: genetic diversity and the success of aquatic invasions. Trends in Ecology \& Evolution, 22, 454-464.

Rius M, Darling JA (2014) How important is intraspecific genetic admixture to the success of colonising populations? Trends in Ecology \& Evolution, 29, 233-242.

Saleh MA, \& Basuony M (1998) A contribution to the mammalogy of the Sinai Peninsula. Mammalia, 62, 557-575.

Schmid-Hempel P, Schmid-Hempel R, Brunner PC, Seeman OD, Allen GR (2007) Invasion success of the bumblebee, Bombus terrestris, despite a drastic genetic bottleneck. Heredity, 99, 414-422.

Shine R, Brown GP, Phillips BL (2011) An evolutionary process that assembles phenotypes through space rather than through time. Proceedings of the National Academy of Sciences, 108, 57085711.

Signorile AL, Wang J, Lurz PWW et al. (2014) Do founder size, genetic diversity and structure influence rates of expansion of North American grey squirrels in Europe? Diversity and Distributions, 20, 918-930.

Slate J, Pemberton JM (2002) Comparing molecular measures for detecting inbreeding depression. Journal of Evolutionary Biology, 15, 20-31.

Stamatakis A (2006) RAxML-VI-HPC: maximum likelihood-based phylogenetic analyses with thousands of taxa and mixed models. Bioinformatics, 22, 2688-2690.

Strayer DL, Eviner VT, Jeschke JM, Pace ML (2006) 
bioRxiv preprint doi: https://doi.org/10.1101/016493; this version posted April 23, 2016. The copyright holder for this preprint (which was

not certified by peer review) is the author/funder, who has granted bioRxiv a license to display the preprint in perpetuity. It is made available under aCC-BY-NC-ND 4.0 International license.

Understanding the long-term effects of species invasions. Trends in Ecology \& Evolution, 21, 645651.

Tayeh A, Estoup A, Hufbauer RA et al. (2013) Investigating the genetic load of an emblematic invasive species: the case of the invasive harlequin ladybird Harmonia axyridis. Ecology and Evolution, 3, 864-871.

Thuiller W, Broennimann O, Hughes G et al. (2006) Vulnerability of African mammals to anthropogenic climate change under conservative land transformation assumptions. Global Change Biology, 12, 424-440.

Trucchi E, Gratton P, Whittington JD et al. (2014) King penguin demography since the last glaciation inferred from genome-wide data. Proceedings of the Royal Society B: Biological Sciences, 281, 20140528.

Trucchi E, Sbordoni V (2009) Unveiling an ancient biological invasion: molecular analysis of an old European alien, the crested porcupine (Hystrix cristata). BMC Evolutionary Biology, 9, 109.

Vandepitte K, de Meyer T, Helsen K et al. (2014) Rapid genetic adaptation precedes the spread of an exotic plant species. Molecular Ecology, 23, 21572164.

Wagner CE, Keller I, Wittwer S et al. (2013) Genomewide RAD sequence data provide unprecedented resolution of species boundaries and relationships in the Lake Victoria cichlid adaptive radiation. Molecular Ecology, 22, 787-798.

Weir BJ (1974) Reproductive characteristics of Hystricomorph Rodents. Symposia of the Zoological Society of London, 34, 265-301.

White TA, Perkins SE, Heckel G, Searle JB (2013) Adaptive evolution during an ongoing range expansion: the invasive bank vole (Myodes glareolus) in Ireland. Molecular Ecology, 22, 29712985.

Zayed A, Constantin ŞA, Packer L (2007) Successful biological invasion despite a severe genetic load. PLOS ONE, 2, e868. 
bioRxiv preprint doi: https://doi.org/10.1101/016493; this version posted April 23, 2016. The copyright holder for this preprint (which was not certified by peer review) is the author/funder, who has granted bioRxiv a license to display the preprint in perpetuity. It is made available under aCC-BY-NC-ND 4.0 International license.

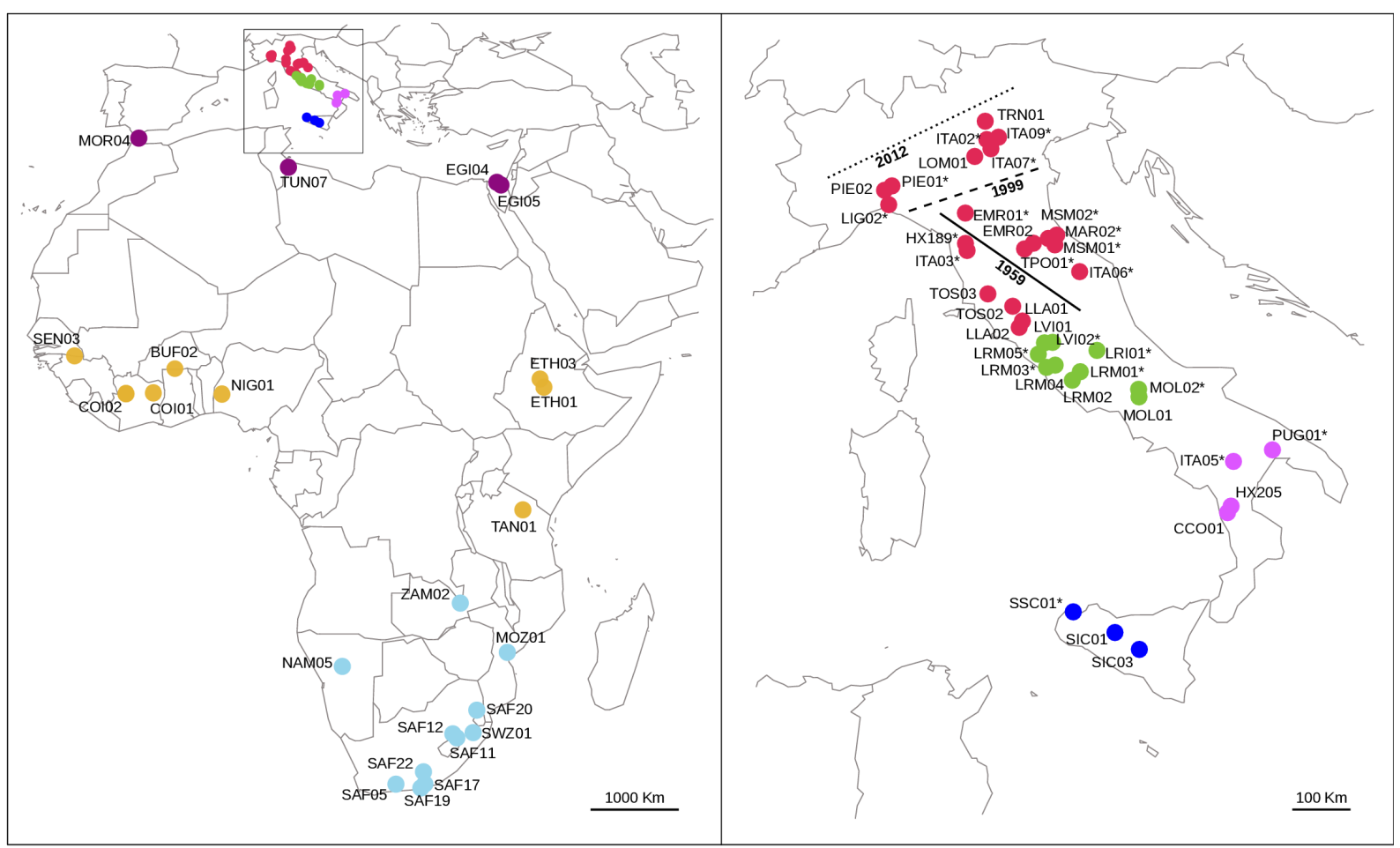

Figure 1. Distribution of the genotyped individuals in Africa (left panel) and Italy (right panel). Colors identify a priori groups defined in the text: H.afr (light blue), H.cri-SS (orange), H.cri-NA (purple), H.cri-IT-Sicily (blue), H.cri-IT-south (light purple), H.cri-IT-centre (green), H.cri-IT-north (red). The recent range expansion documented in North Italy is also shown: the black solid line represents the north-easternmost distribution in 1959 (H.cri-IT-north-1959); the large-dashed black line represents the northernmost distribution in 1999 (H.cri-IT-north-1999) and the small-dashed black line represents the northernmost distribution in 2012 (H.cri-IT-north-2012) according to Angelici \& Amori (1999) and Mori et al. (2013). Individuals from Italy included in both the global and the invasive_reduced dataset are not marked with an asterisk; in the text we refer to this group of samples as H.cri-IT. 
bioRxiv preprint doi: https://doi.org/10.1101/016493; this version posted April 23, 2016. The copyright holder for this preprint (which was

not certified by peer review) is the author/funder, who has granted bioRxiv a license to display the preprint in perpetuity. It is made available under aCC-BY-NC-ND 4.0 International license.

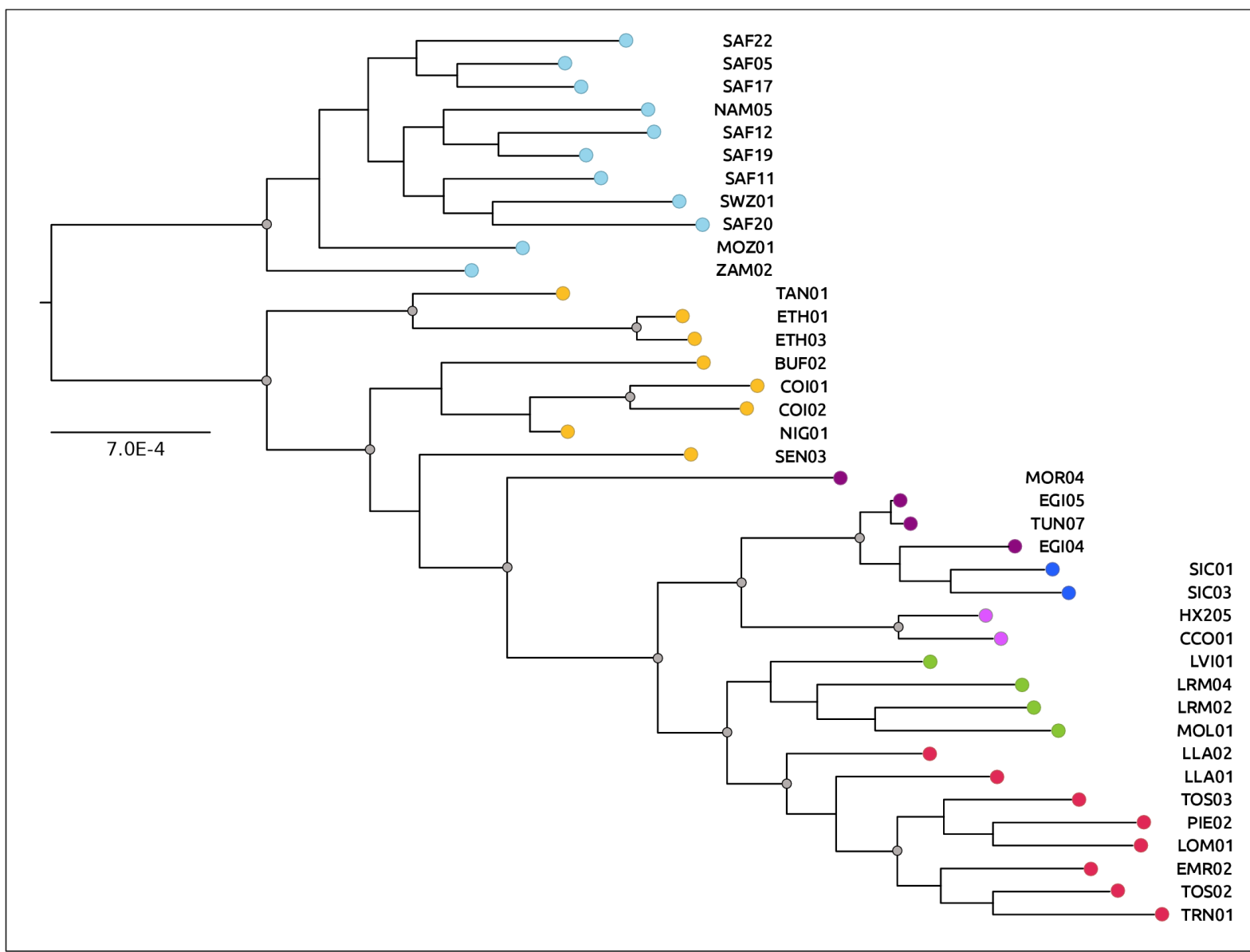

Figure 2. Global genetic structure. Maximum-Likelihood tree built using the global dataset (all individuals not marked with an asterisk in Africa and Italy in Fig.1). Nodes with bootstrap support of $>99 \%$ are shown (grey filled circles). Colors represent the same geographic locations as in Fig. 1. 
bioRxiv preprint doi: https://doi.org/10.1101/016493; this version posted April 23, 2016. The copyright holder for this preprint (which was

not certified by peer review) is the author/funder, who has granted bioRxiv a license to display the preprint in perpetuity. It is made available under aCC-BY-NC-ND 4.0 International license.

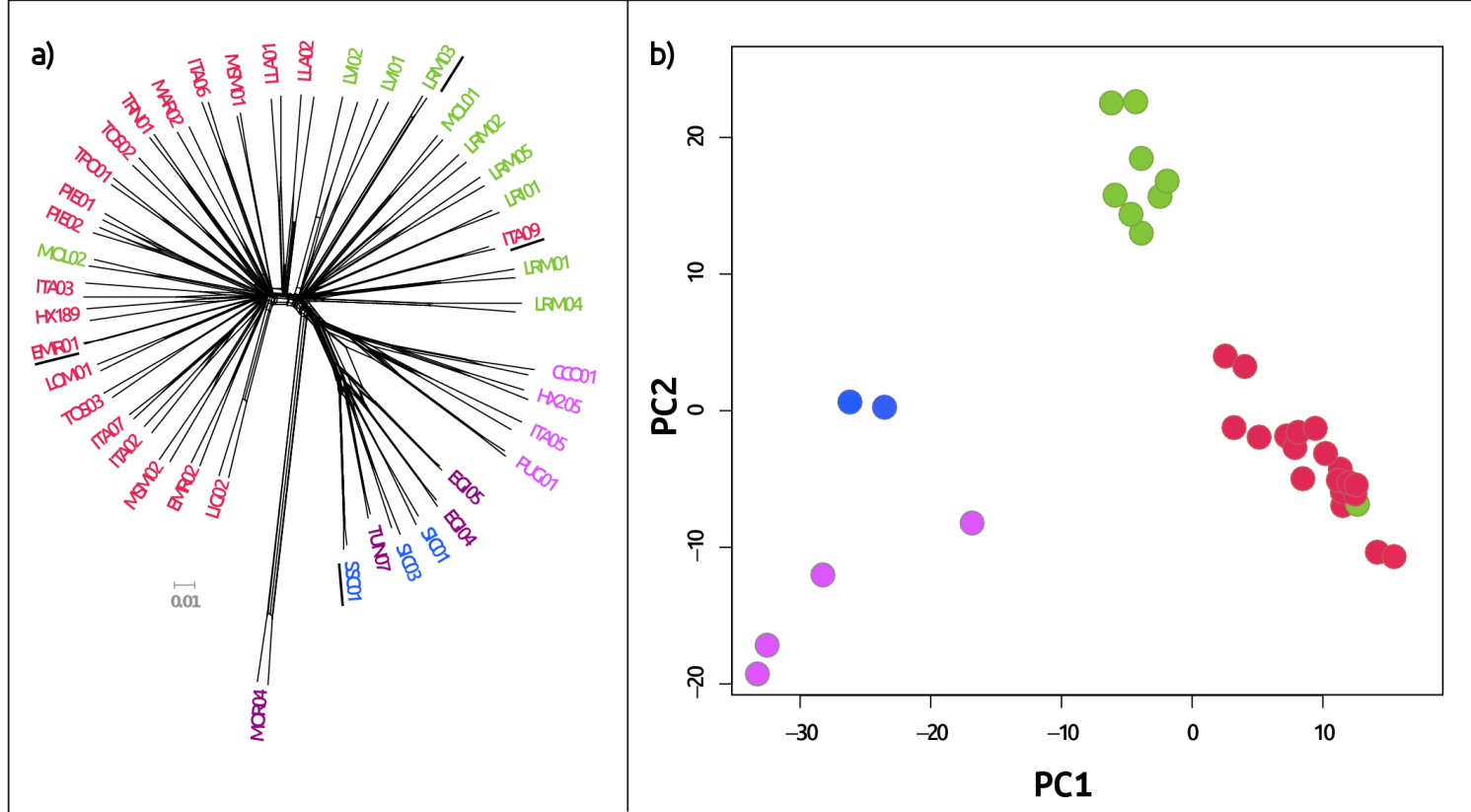

Figure 3. Structure and diversity in the invasive range. Neighbor-Joining Network (a) and principal component analysis (b) using the invasive dataset (See Methods for details). Some individuals (EMR01, ITA09, SSC01, LRM03; underlined in panel a) are included only in the Neighbor-Joining Network but not in the PCA; they were discarded according to the strict filtering required for this analysis. Colors represent the same geographic locations as in Fig. 1. 
bioRxiv preprint doi: https://doi.org/10.1101/016493; this version posted April 23, 2016. The copyright holder for this preprint (which was

not certified by peer review) is the author/funder, who has granted bioRxiv a license to display the preprint in perpetuity. It is made available under aCC-BY-NC-ND 4.0 International license.

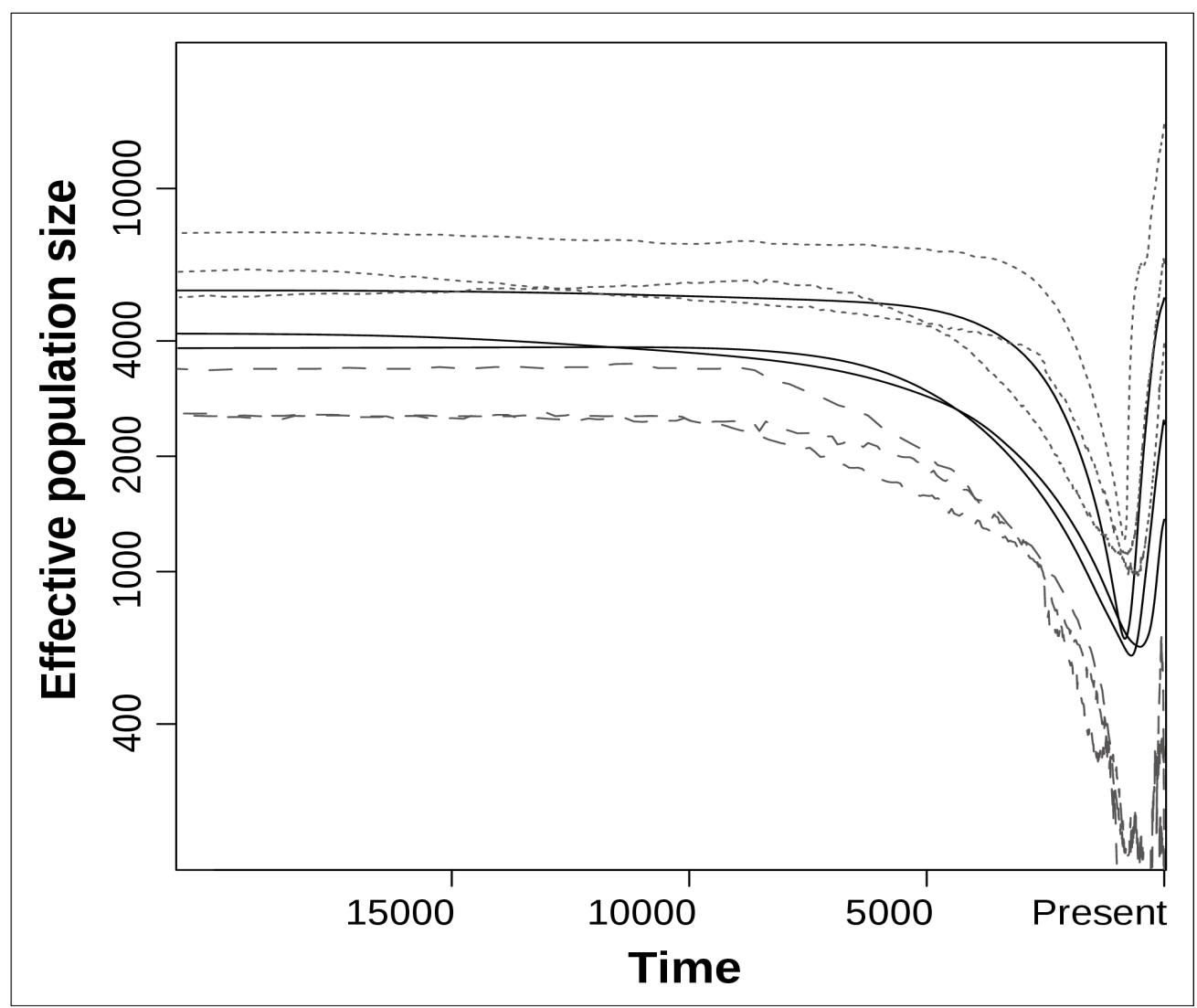

Figure 4. Recent demography of the invasive population. Extended Bayesian Skyline plot using 16 selected individuals from the invasive range not marked with an asterisk in Fig.1 (invasive_reduced dataset; see Methods for details). Results of three independent runs including $50 \mathrm{RAD}$ loci and one mitochondrial locus are shown. The median (black), as well as the lower (gray large-dashed) and the upper (gray small-dashed) boundaries of the 95\% credible region are shown. Time is shown in years before present (x-axis) while effective population size is in individuals (y-axis). 
bioRxiv preprint doi: https://doi.org/10.1101/016493; this version posted April 23, 2016. The copyright holder for this preprint (which was

not certified by peer review) is the author/funder, who has granted bioRxiv a license to display the preprint in perpetuity. It is made available under aCC-BY-NC-ND 4.0 International license.
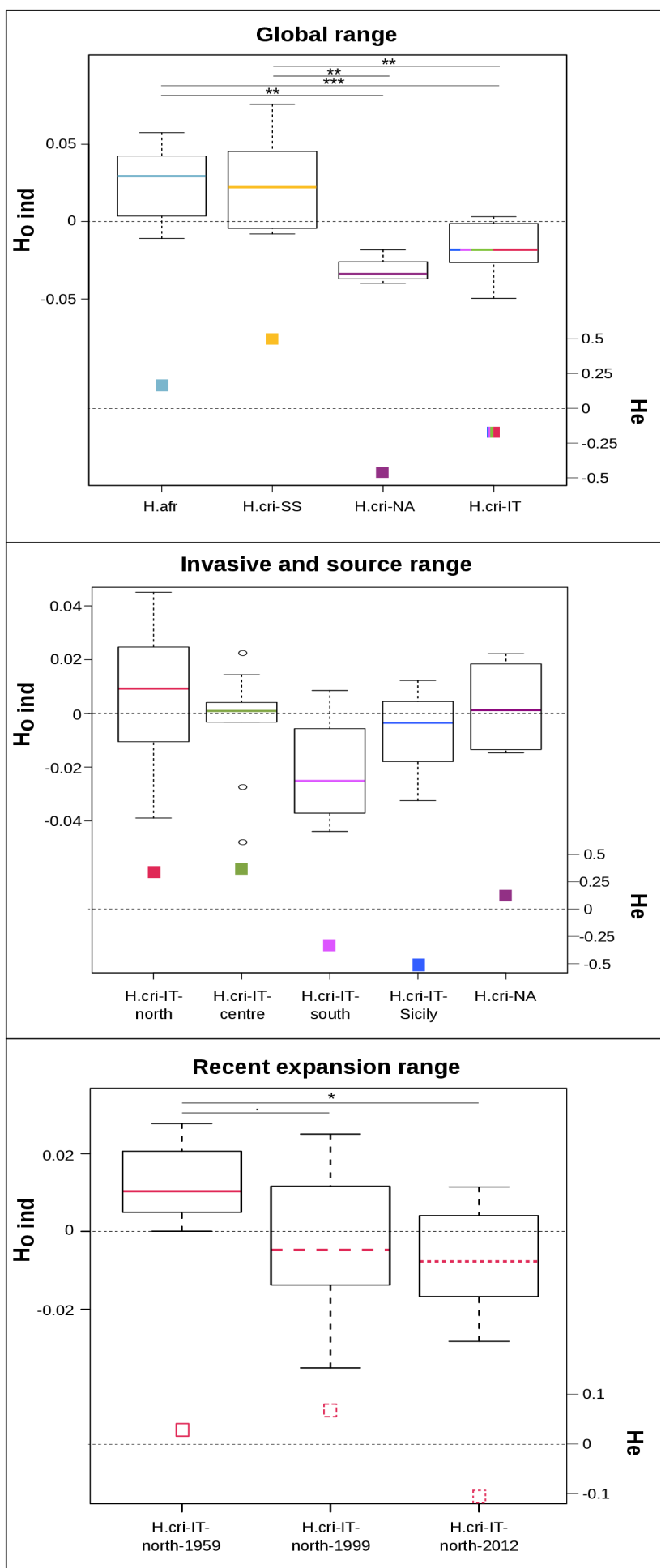

Figure 5. Observed and expected heterozygosity in native and invasive populations. Residuals of the model $\mathrm{H}_{\mathrm{o} \text { ind }} \sim \log (\mathrm{raw}$ reads) and $\mathrm{H}_{\mathrm{e}}$ as deviation from the mean are analyzed across the native and invasive range $(\mathrm{p}<0.001$ considering all comparisons) using the global dataset (a), across the invasive and the source native range $(\mathrm{p}=0.237$ considering all comparisons) using the invasive dataset (b), and across the recent expansion range ( $\mathrm{p}=0.058$ considering all comparisons) using the invasive dataset (c). Pairwise significance between populations/groups is reported only in case the p-value for the geographic factor across all comparisons was below 0.1 (a and c panels). P-value pairwise: '.' < 0.1; '*' < 0.05; '**' < 0.01 ; ${ }^{\prime * * * '}<0.001$. Colors and patterns represent the same geographic locations as in Fig. 1. 